\title{
Newcastle disease conjunctivitis with subepithelial infiltrates
}

\author{
ROBERT H. HALES AND H. BRUGE OSTLER \\ From the Department of Ophthalmology and the Francis I. Proctor Foundation for Research in \\ Ophthalmology, University of California, San Francisco, U.S.A.
}

An epidemic of Newcastle disease occurred in turkeys of Southern Utah in the late fall of 1965. Several workers in intimate contact with the turkeys at the processing plant developed a follicular conjunctivitis subsequently identified as due to Newcastle disease virus. The typical clinical findings and rise in haemagglutinin-inhibition titre indicated that the infection was acquired from the turkeys during the processing.

Newcastle disease conjunctivitis in humans is usually a moderately severe follicular conjunctivitis with involvement of the preauricular nodes. The cornea is rarely affected (Allen, Burns, Gingrich, Givner, Key, Kimura, and Thygeson, 1963; Allen, 1964; Trott and Pilsworth, 1965), although superficial infiltrates and epithelial staining have been reported (Keeney and Hunter, I950; Jones, 1962). To our knowledge, subepithelial infiltrates have not previously been described.

\section{Case reports}

Case 1, a 42-year-old white female, was first seen on November 26, 1965. She had had a watery discharge and redness of the left eye for 5 days and of the right eye for 1 day. When she was first seen the eyes had not been treated.

\section{Examination}

The uncorrected visual acuity was $20 / 20, \mathrm{~J}_{2}$ in both eyes. The preauricular nodes were tender and grossly visible on both sides. There was follicular and papillary hypertrophy of the upper and lower tarsal conjunctivae of both eyes, most marked inferiorly. Both corneae showed some superficial punctate staining of the exposed areas, and corneal sensation was slightly diminished.

Scrapings from both the upper and lower tarsi showed a predominantly mononuclear leucocytic reaction, a few normal and a few degenerating epithelial cells, and some mucus. No pathogenic organisms grew in blood agar cultures of conjunctival material.

\section{Treatment}

The eyes were treated topically with sodium sulphacetamide and healed in about a week.

Case 2, a 13-year-old white boy, was first seen on January 7, ig66. One eye had been red for 2 days and there was a watery, mucoid discharge. For 4 days he had been treated with penicillin for tonsillitis. There was a history of recurrent styes. A member of his family worked at the turkey plant.

\section{Examination}

The uncorrected visual acuity was $20 / 50$ in the right eye and 20/20 in the left. There was marked injection and oedema of the lids and bulbar conjunctivae and the preauricular nodes were tender 
and grossly visible. The anterior cervical nodes were also enlarged. Small subconjunctival haemorrhages, associated with a mild follicular and papillary conjunctival hypertrophy, were noted in the right eye, and there was marked follicular and papillary hypertrophy of the inferior tarsal conjunctiva of both eyes. Both corneae showed areas of superficial punctate staining in the exposed areas.

Bacterial cultures showed no growth. Conjunctival scrapings from the right conjunctiva contained many mononuclear leucocytes, some polymorphonuclear leucocytes, and normal epithelial cells. Tests of acute and convalescent sera showed a rise in the haemagglutinin-inhibition titre for Newcastle disease antibodies from zero to $1: 10^{*}$. The patient was given systemic penicillin and topical sulphacetamide.

\section{Progress}

After Io weeks, the patient stated that the conjunctivitis had become bilateral and had persisted for a week. The visual acuity had not changed and corneal sensation was normal. Distinct paracentral subepithelial infiltrates with a definitely granular appearance were now visible with the slit lamp (Figure). The corneae no longer stained, but the infiltrates were in the areas of the superficial staining previously noted. Gradually, over a period of 18 months, the subepithelial lesions disappeared.

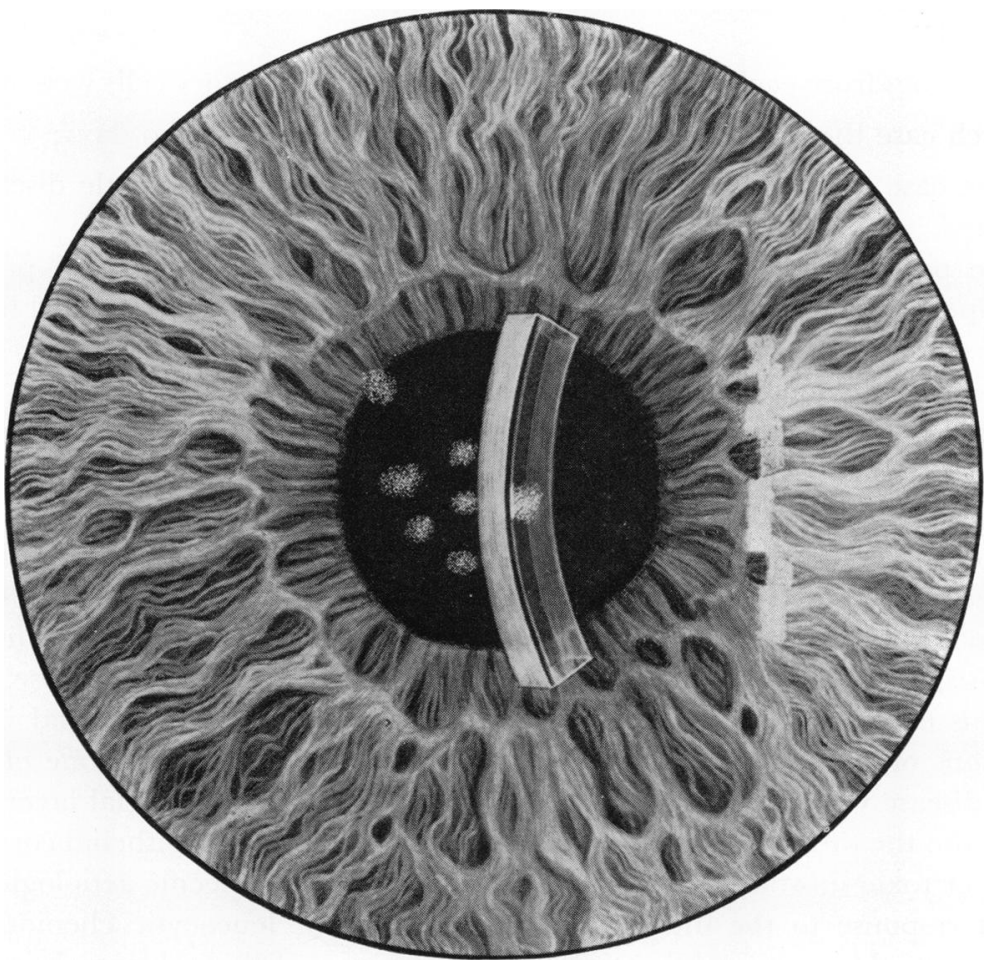

FIGURE Drawing of corneal lesions

Case 3, a 32-year-old white male turkey processor, was first seen on November 26, ig66. He had a 5-day history of bilateral conjunctivitis accompanied by a watery discharge and tender preauricular nodes. He had been treated with polymyxin B, sulphate-zinc, bacitracin (Polysporin ${ }^{\mathbf{R}}$ ), topical sodium sulphacetamide, and systemic demeclocycline hydrochloride (Declomycin ${ }^{\mathbf{R}}$ ) without effect. No systemic illness was noted. The patient's wife, who had also been working with turkeys and had a severe follicular conjunctivitis, was not examined by the authors.

* Test performed by Utah State Department of Health Bureau of Laboratories. 


\section{Examination}

The uncorrected visual acuity was $20 / 20, \mathrm{~J}_{1}$, in both eyes,. Both follicular and papillary hypertrophy were noted over the upper tarsal conjunctivae, and there was papillary hypertrophy of the lower tarsal conjunctivae. The corneae of both eyes showed many superficial punctate staining areas. There were no subepithelial infiltrates. Corneal sensation was slightly diminished.

No pathogenic organisms grew in conjunctival cultures. Conjunctival scrapings contained a few mononuclear leucocytes, a few polymorphonuclear leucocytes, and normal epithelial cells, but there were no inclusions, organisms, or eosinophils.

The patient did not return for re-examination.

\section{Comment}

Newcastle disease virus was probably the cause of the conjunctivitis in these three cases-a conclusion based on the following observations:

(I) The conjunctivitis occurred in people associated with the turkey processing plant or working directly with the turkeys;

(2) It occurred coincidentally with a known epidemic of Newcastle disease in the turkeys;

(3) In each case the disease was follicular in type and associated with an enlarged preauricular node;

(4) In scrapings from each case the predominating inflammatory cells were mononuclear;

(5) In each case the conjunctival disease persisted for less than ro days;

(6) In one case a rise in haemagglutinin-inhibition titre to Newcastle disease virus was demonstrated.

All three patients had bilateral follicular conjunctivitis that affected principally the inferior palpebral conjunctiva. Marked chemosis and a few subconjunctival haemorrhages suggested severe disease. In each case superficial punctate staining of the exposed area of the cornea was demonstrable, and in one there were subepithelial infiltrates that resolved over a period of I 8 months. Tender, grossly visible preauricular nodes were consistently present.

It is significant that both punctate keratitis and epithelial infiltrates have been observed in this disease (Keeney and Hunter, I950; Thygeson, 1952). Subepithelial infiltrates are normally associated with epidemic kerato-conjunctivitis (Laibson, Dhiri, Oconer, and Ortolan, 1970), and have also been found on occasion in other types of follicular keratoconjunctivitis, as for example, inclusion conjunctivitis (Dawson, Wood, Rose, and Hanna, 1967). This experience implies that epithelial keratitis and subepithelial infiltrates are manifestations of the same disease process, and that the infiltrates in one of our cases of Newcastle disease were the result of virus extension to the subepithelial layers.

There is also the theory (Aronson and Moore, I 969) that a subepithelial corneal response to immune or toxic substances is not a direct response to the specific aetiological agent but an indirect response to the induced polymorphonuclear leucocytic chemotaxis and degranulation. If this is so, stabilization of the lysozymes of the polymorphonuclear leucocytes with corticosteroid therapy should prevent subepithelial infiltration. This alternative theory is supported by the fact that the subepithelial infiltrates of epidemic keratoconjunctivitis can be reduced with corticosteroid therapy (Laibson and others, 1967).

\section{Summary}

Three cases of Newcastle disease conjunctivitis are presented; one patient with a rising 
antibody titre developed subepithelial infiltrates, which disappeared over an I8-month observation period. All three patients showed grossly visible, tender preauricular nodes, both follicular and papillary conjunctival hypertrophy, and superficial punctate corneal staining.

Newcastle disease conjunctivitis should be considered in the differential diagnosis of follicular conjunctivitis complicated by epithelial keratitis and subepithelial infiltrates.

\section{References}

AlleN, H. F., BURNS, R. P., GINGRIGH, W. D., GIVNER, I., KEY, S. N., JR., KIMURA, S. J., and THYGESON, P.

(1963) "Infectious Diseases of the Conjunctiva and Cornea: Symposium of the New Orleans Academy of Ophthalmology", p. 107. Mosby, St. Louis

AlleN, J. H., (1964) Int. Ophthal. Clin., 4, $3^{1} \mathrm{I}$

ARonson, s. B., and moore, t. E., JR. (1969) Amer. J. Ophthal., 67, 873

DAwson, C., WOOD, T. R., ROSE, L., and HANNA, L. (I967) Arch. Ophthal. (Chicago), 78, 34 I

JONES, B. R. (I962) "Differential diagnosis of punctate keratitis", in "International Ophthalmology

Clinics", No. 3. Diseases of the Cornea, ed. P. D. Trevor-Roper, vol. 2, p. 591. Little Brown, Boston

KEENEY, A. H., and HUNTER, M. G. (1950) Arch. Ophthal. (Chicago), 44, 573

LAIBSON, P. R., DHIRI, s., Oconer, J., and ORTOLAN, G. (I970) Ibid., 84, 36

THYGeson, P., (I952) in "Progress in Ophthalmology and Otolaryngology", vol. I, ed. M. Weiner,

A. E. Manmenee, P. E. Ireland, and J. A. Sullivan, p. I4. Grune and Stratton, New York TROTT, D. G., and PILSWORTH, R. (1965) Brit. med. F., 2, I5 14 